Tze Ping Loh*, Andrea Rita Horvath, Cheng-Bin Wang, David Koch, Giuseppe Lippi, Nicasio Mancini, Maurizio Ferrari, Robert Hawkins, Sunil Sethi and Khosrow Adeli, The International Federation of Clinical Chemistry and Laboratory Medicine Taskforce on COVID-19

\title{
Laboratory practices to mitigate biohazard risks during the COVID-19 outbreak: an IFCC global
}

\section{survey}

https://doi.org/10.1515/cclm-2020-0711

Received May 13, 2020; accepted May 14, 2020

\section{Abstract}

Objectives: A global survey was conducted by the IFCC Task Force on COVID-19 to better understand how general biochemistry laboratories manage the pre-analytical, analytical and post-analytical processes to mitigate biohazard risks during the coronavirus disease 2019 (COVID-19) pandemic.

Methods: An electronic survey was developed to record the general characteristics of the laboratory, as well as the pre-analytical, analytical, post-analytical and operational practices of biochemistry laboratories that are managing clinical samples of patients with COVID-19.

Results: A total of 1210 submissions were included in the analysis. The majority of responses came from hospital central/core laboratories that serve hospital patient groups and handle moderate daily sample volumes. There

*Corresponding author: Tze Ping Loh, Department of Laboratory Medicine, National University Hospital, 5 Lower Kent Ridge Road, Singapore 119074, Singapore, Phone: (+65) 67724345, Fax: (+65) 67771613, E-mail: tploh@hotmail.com

Andrea Rita Horvath: Department of Clinical Chemistry and Endocrinology, New South Wales Health Pathology, Prince of Wales Hospital, Sydney, Australia

Cheng-Bin Wang: Department of Laboratory Medicine, Chinese PLA General Hospital, Beijing, P.R. China

David Koch: Department of Pathology and Laboratory Medicine, Emory University, Atlanta, GA, USA

Giuseppe Lippi: Section of Clinical Biochemistry, Department of Neuroscience, Biomedicine and Movement, University of Verona, Verona, Italy

Nicasio Mancini: Laboratory of Microbiology and Virology, University Vita-Salute San Raffaele, Milan, Italy; and IRCCS San Raffaele Hospital, Milan, Italy

Maurizio Ferrari: Università Vita-Salute San Raffaele, Milan, Italy

Robert Hawkins: Department of Laboratory Medicine, Tan Tock Seng Hospital, Singapore, Singapore

Sunil Sethi: Department of Laboratory Medicine, National University Hospital, Singapore, Singapore

Khosrow Adeli: Clinical Biochemistry, The Hospital for Sick Children, University of Toronto, Toronto, ON, Canada has been a decrease in the use of pneumatic tube transport, increase in hand delivery and increase in number of layers of plastic bags for samples of patients with clinically suspected or confirmed COVID-19. Surgical face masks and gloves are the most commonly used personal protective equipment (PPE). Just $>50 \%$ of the laboratories did not perform an additional decontamination step on the instrument after analysis of samples from patients with clinically suspected or confirmed COVID-19. A fifth of laboratories disallowed add-on testing on these samples. Less than a quarter of laboratories autoclaved their samples prior to disposal.

Conclusions: The survey responses showed wide variation in pre-analytical, analytical and post-analytical practices in terms of PPE adoption and biosafety processes. It is likely that many of the suboptimal biosafety practices are related to practical local factors, such as limited PPE availability and lack of automated instrumentation.

Keywords: biohazard; biosafety; COVID-19; SARS-CoV-2.

\section{Introduction}

Since December 2019, an outbreak caused by a novel coronavirus called SARS-CoV-2 (severe acute respiratory syndrome coronavirus 2) and causing the coronavirus disease 2019 (COVID-19) has emerged in China and has then spread inside and outside the country [1]. On 11 March 2020, the outbreak was declared a pandemic by the World Health Organization in view of its rapid global spread [2]. COVID19 has now affected virtually all countries worldwide. Laboratory medicine is one of the most important front-line professions assisting the fight against the pandemic, by producing test results that are crucial for the diagnosis and management of COVID-19 patients [3, 4]. Therefore, due to the frequent contact with potentially infected biological material, it is crucial to ensure that laboratory processes remain safe and effective, and that laboratory test results can be always timely delivered during the pandemic. 
There are few professional biosafety guidelines specifically focused on clinical laboratories, i.e. those with a general biosafety level $\leq 2[5-7]$. Nonetheless, there are important risks connected with some laboratory activities when performing analysis on uncapped clinical specimens, including the need to dispense, vortex and centrifuge clinical samples, and the need to manually handle samples on an open bench [8]. These procedures carry a risk of aerosolization, which is a minor but not meaningless source of potential SARS-CoV-2 contagion. Additionally, the high volume of samples and short turnaround time required for some critical test results put considerable pressure on the laboratory to adopt enhanced biosafety precautions without significantly compromising efficiency.

The study of viral kinetic has demonstrated that the highest viral load is certainly detectable in respiratory samples. However, other samples may harbor high viral loads even if at a lower rate, including those most commonly processed in a clinical laboratory, such as stool and, more importantly, serum or plasma. As an example, a recent study detected SARS-CoV-2 in 41\% of COVID-19 patients up to 30 days after the symptom onset, even if with an approximately 100 -fold lower load compared to respiratory samples [9]. Importantly, only one single patient (1\%) in this study featured detectable virus in urine.

The International Federation of Clinical Chemistry and Laboratory Medicine (IFCC) Task Force on COVID-19 has been established with one of the main objectives being to recommend biosafety measures while handling clinical samples during the COVID-19 pandemic (https://www.ifcc.org/executive-board-and-council/ebtask-forces/ifcc-task-force-on-covid-19/). A global survey has hence been planned and conducted by the Task Force for better understanding how clinical laboratories manage the pre-analytical, analytical and post-analytical processes, with the final aim to have a clear picture of the current situation and develop harmonized indications for mitigating biohazard risks during the COVID-19 outbreak. This article describes the first part of data garnered with this survey; the latter being published in a separate companion article [10].

\section{Materials and methods}

A survey questionnaire has been originally developed by three chemical pathologists from Singapore and Australia (see Supplemental Material). A brief introduction explained the intent of the survey. An implied consent was sought from survey participants to publish the information provided, including making the conclusions of the survey publicly accessible, thereby allowing laboratories to evaluate their own local practices and compare those to peers all around the globe. No individually identifiable information was solicited from participants.

Following this, survey questions were designed to record the general characteristics of the laboratory, as well as the pre-analytical, analytical, post-analytical and operational practices of facilities which are managing clinical samples of patients with COVID-19. The initial draft questionnaire was pilot tested in seven laboratories in Singapore and Sydney (Australia) for content and language, and underwent two cycles of refinements.

The survey questionnaire was subsequently built into an enterprise internet survey engine (Verint Systems, Melville, NY, USA). The electronic survey was initially published in English on April 16, 2020, with Chinese, Spanish and French translations made available on April 22, 2020. The survey was publicized through multiple channels, including the IFCC eNewsFlash, the Canadian Society for Clinical Chemistry, the Australasian Association for Clinical Biochemistry and Laboratory Medicine, the European Federation of Clinical Chemistry and Laboratory Medicine, American Association for Clinical Chemistry, the Spanish Society of Laboratory Medicine, Korean Society for Clinical Chemistry, Chinese Society of Laboratory Medicine, Argentinian National Society and the Latin American Confederation of Clinical Biochemistry. The survey was closed on May 1, 2020.

The survey responses were exported from the survey engine into an Excel file and analyzed using Analyse-It (for Microsoft Excel, Microsoft, Seattle, WA, USA). This study focused on the survey responses related to pre-analytical, analytical and post-analytical processes and were summarized using descriptive statistics.

\section{Results}

The survey received 1483 submissions until the deadline, and 273 submissions were excluded from analysis as the laboratories were either not managing clinical samples from patients with COVID-19 at the time of the survey, or did not indicate so. A total of 1210 replies were hence included in the final analysis, 921 of which were in English, 62 in Chinese, 183 in Spanish and 44 in French. Submissions were received from 86 countries and regions, providing a good global representation. The countries and regions of practice of participants are summarized in Supplemental Table 1. The majority of responses came from hospital central/core laboratories serving inpatient/ outpatient/intensive care units/emergency department patient groups that handle moderate volume of daily samples. Most laboratories used some form of laboratory automation. The general characteristics of survey participants are summarized in Table 1.

\section{Pre-analytical processes}

The responses to the questions regarding pre-analytical processes during the COVID-19 outbreak are summarized 
Table 1: General characteristics of survey participants.

\begin{tabular}{|c|c|c|}
\hline Question & Response & $\begin{array}{r}\text { Frequency (\% of } \\
\text { total submission) }\end{array}$ \\
\hline \multirow{4}{*}{$\begin{array}{l}\text { Which of the following best describes } \\
\text { your biochemistry laboratory? }\end{array}$} & Hospital central/core laboratory & $998(82.4)$ \\
\hline & Hospital satellite laboratory & $69(5.7)$ \\
\hline & Community-based laboratory & $81(6.7)$ \\
\hline & Other & $62(5.1)$ \\
\hline \multirow{6}{*}{$\begin{array}{l}\text { Which of the following patient } \\
\text { populations are served by your } \\
\text { biochemistry laboratory? Please select } \\
\text { all that apply. }\end{array}$} & Inpatient & $1101(90.1)$ \\
\hline & Outpatient & $1085(89.7)$ \\
\hline & Emergency department & $1023(84.5)$ \\
\hline & Intensive care units & $1007(83.2)$ \\
\hline & Other & $171(14.1)$ \\
\hline & Not answered & $4(0.3)$ \\
\hline \multirow{5}{*}{$\begin{array}{l}\text { Please indicate the number of patient } \\
\text { samples your laboratory processes per } \\
\text { day? }\end{array}$} & $<200$ samples & $301(24.9)$ \\
\hline & 200-999 samples & $446(36.9)$ \\
\hline & 1000-2500 samples & $253(20.9)$ \\
\hline & $>2500$ samples & $204(16.9)$ \\
\hline & Not answered & $6(0.5)$ \\
\hline \multirow{8}{*}{$\begin{array}{l}\text { Does the biochemistry laboratory use } \\
\text { any of the following components of a } \\
\text { laboratory automation system? Please } \\
\text { select all that apply. }\end{array}$} & Centrifugation & $752(20.3)$ \\
\hline & Decapping & $652(53.9)$ \\
\hline & Aliquoting & $594(49.1)$ \\
\hline & Recapping & $508(42.0)$ \\
\hline & Sample storage & $593(49.0)$ \\
\hline & Sample conveyance track & $466(38.5)$ \\
\hline & $\begin{array}{l}\text { We do not use a laboratory } \\
\text { automation system }\end{array}$ & $373(30.8)$ \\
\hline & Not answered & $12(1.0)$ \\
\hline
\end{tabular}

in Figure 1. In nearly half of clinical laboratories, samples from patients with clinically suspected or confirmed COVID-19 were marked with a special label. In patients with clinically suspected and confirmed COVID$19,83.4 \%$ and $75 \%$ of the laboratory blood samples were delivered by hand, respectively. The use of pneumatic tube system for sample transportation was reduced. There was an increase in the use of double and triple layers of plastic (sample/kangaroo) bags for transporting samples from patients with clinically suspected or confirmed COVID-19. A third of laboratories required sample bags to be placed in a solid-walled, leak proof container.

Surgical face masks and gloves were the two most common pieces of personal protection equipment (PPE) used when manually handling pre-analytical processes such as sample reception, centrifugation, aliquoting and decapping. Just less than half of laboratories used goggles or face shields, a third used disposable gowns and only a quarter used N95 (or equivalent) face masks. Class II biosafety cabinets were only used in one fifth of laboratories for sample aliquoting and decapping, and the cabinets were used by one tenth of the laboratories when receiving and centrifuging the samples. Less than $4 \%$ of laboratories used a negative pressure room for handling clinical specimens from clinically suspected or confirmed patients with COVID-19.

\section{Analytical processes}

The responses to the questions regarding analytical processes during the COVID-19 outbreak are summarized in Figure 2. The use of PPE in the analytical phase, such as during dilution, extraction and vortexing, was marginally lower than in the pre-analytical phase. Fewer laboratories reported use of PPE when testing patient samples on point-of-care analyzers. By contrast, more laboratories used surgical face masks and gloves when manually handling urine samples.

Just more than half of the laboratories did not perform an additional decontamination step on the analyzers after 
Pre-analytical

Question: Are samples of the following patients indicated with a special label (e.g. sticker or hand-written symbol or label)?

$\begin{array}{lrr} & \text { Patients with clinically } & \text { Patients with } \\ \text { Yes } & \text { suspected COVID-19 } & \text { confirmed COVID-19 } \\ \text { No } & 675(55.8) & 582(48.1) \\ \text { Not answered } & 504(41.7) & 458(37.9) \\ & 31(2.6) & 170(14.0)\end{array}$

Question: How are biochemistry samples delivered to your laboratory ? Please select all that apply.

$\begin{array}{lrrr} & \text { Routine } & \text { Patients with clinically } & \text { Patients with } \\ & \text { patients } & \text { suspected COVID-19 } & \text { confirmed COVID-19 } \\ \text { By hand } & 937(77.4) & 1009(83.4) & 906(74.9) \\ \text { By pneumatic tube } & 499(37.1) & 309(25.5) & 273(22.6) \\ \text { By motorized vehicle (motorcycle, car, etc) } & 368(30.4) & 277(22.9) & 249(20.6) \\ \text { Other } & 51(4.2) & 53(4.4) & 52(4.3) \\ \text { Not answered } & 41(3.4) & 35(2.9) & 153(12.6)\end{array}$

Question: How are samples packed for delivery to your laboratory? Please select all that apply.

$\begin{array}{lrrr} & \text { Routine } & \text { Patients with clinically } & \text { Patients with } \\ \text { patient } & \text { suspected COVID-19 } & \text { confirmed COVID-19 } \\ \text { In a single plastic (sample/kangaroo) bag } & 758(62.6) & 405(33.5) & 342(28.3) \\ \text { In two layers of plastic (sample/kangaroo) bag } & 91(7.5) & 315(26.0) & 282(23.3) \\ \text { In three layers of plastic (sample/kangaroo) bag } & 32(2.6) & 156(12.9) & 148(12.2) \\ \text { The sample in plastic bag(s) is placed in solid- } & 203(16.8) & 396(32.7) & 366(30.2) \\ \text { walled, leak-proof container } & & & \\ \text { Other } & 119(9.8) & 92(7.6) & 84(6.9) \\ \text { Not answered } & 116(9.6) & 58(4.8) & 230(19.0)\end{array}$

Question: What protection processes do the laboratory staff use when manually performing the following on samples from patients with clinically suspected COVID-19? Please select all that apply.

$\begin{array}{lrrrr} & \text { Sample receipt } & \text { Centrifugation } & \text { Aliquoting } & \text { Decapping } \\ \text { Goggles/face shield } & 502(41.5) & 552(45.6) & 562(46.4) & 563(46.5) \\ \text { Surgical face mask } & 853(70.5) & 804(66.4) & 762(63.0) & 719(59.4) \\ \text { N95 (or equivalent) face mask } & 318(26.3) & 327(27.0) & 333(27.5) & 336(27.8) \\ \text { Gloves } & 1093(90.3) & 1057(87.4) & 1015(83.9) & 1012(83.6) \\ \text { Disposable gowns } & 445(36.8) & 433(35.8) & 427(35.3) & 421(34.8) \\ \text { Disposable shoe cover } & 197(16.3) & 194(16.0) & 185(15.3) & 186(15.4) \\ \text { Class I biosafety cabinet } & 62(5.1) & 61(5.0) & 91(7.5) & 89(7.4) \\ \text { Class Il biosafety cabinet } & 119(9.8) & 128(10.6) & 247(20.4) & 220(18.2) \\ \text { Negative pressure room } & 26(2.1) & 29(2.4) & 35(2.9) & 41(3.4) \\ \text { Other } & 72(6.0) & 85(7.0) & 80(6.6) & 84(6.9) \\ \text { Not answered } & 34(2.8) & 68(5.6) & 123(10.2) & 104(8.6)\end{array}$

Question: What protection processes do the laboratory staff use when manually performing the following on samples from patients with confirmed COVID-19? Please select all that apply

$\begin{array}{lr}\text { Goggles/face shield } & \text { Sample receipt } \\ \text { Surgical face mask } & 536(44.3) \\ \text { N95 (or equivalent) face mask } & 766(63.3) \\ \text { Gloves } & 313(25.9) \\ \text { Disposable gowns } & 1000(82.6) \\ \text { Disposable shoe cover } & 450(37.2) \\ \text { Class I biosafety cabinet } & 208(17.2) \\ \text { Class II biosafety cabinet } & 57(4.7) \\ \text { Negative pressure room } & 143(11.8) \\ \text { Other } & 34(2.8) \\ \text { Not answered } & 55(4.5) \\ \end{array}$

$\begin{array}{rrr}\text { Centrifugation } & \text { Aliquoting } & \text { Decapping } \\ 572(47.3) & 584(48.3) & 585(48.3) \\ 716(59.2) & 675(55.8) & 667(55.1) \\ 307(25.4) & 316(26.1) & 322(26.6) \\ 964(79.7) & 932(77.0) & 925(76.4) \\ 439(36.3) & 441(36.4) & 438(36.2) \\ 202(16.7) & 196(16.2) & 198(16.4) \\ 57(4.7) & 83(6.9) & 79(6.5) \\ 146(12.1) & 250(20.7) & 225(18.6) \\ 34(2.8) & 41(3.4) & 42(3.5) \\ 60(5.0) & 59(4.9) & 61(5.0) \\ 147(12.1) & 175(14.5) & 195(16.1)\end{array}$

Figure 1: Frequency of responses to questions related to pre-analytical processes to mitigate biohazard risks in clinical biochemistry laboratories during the COVID-19 pandemic.

The percentages are expressed as fractions of the total number of valid submissions $(n=1210)$. 
Analytical

Question: What protection processes do the laboratory staff use when manually performing the following on samples from patients with clinically suspected COVID-19? Please select all that apply.

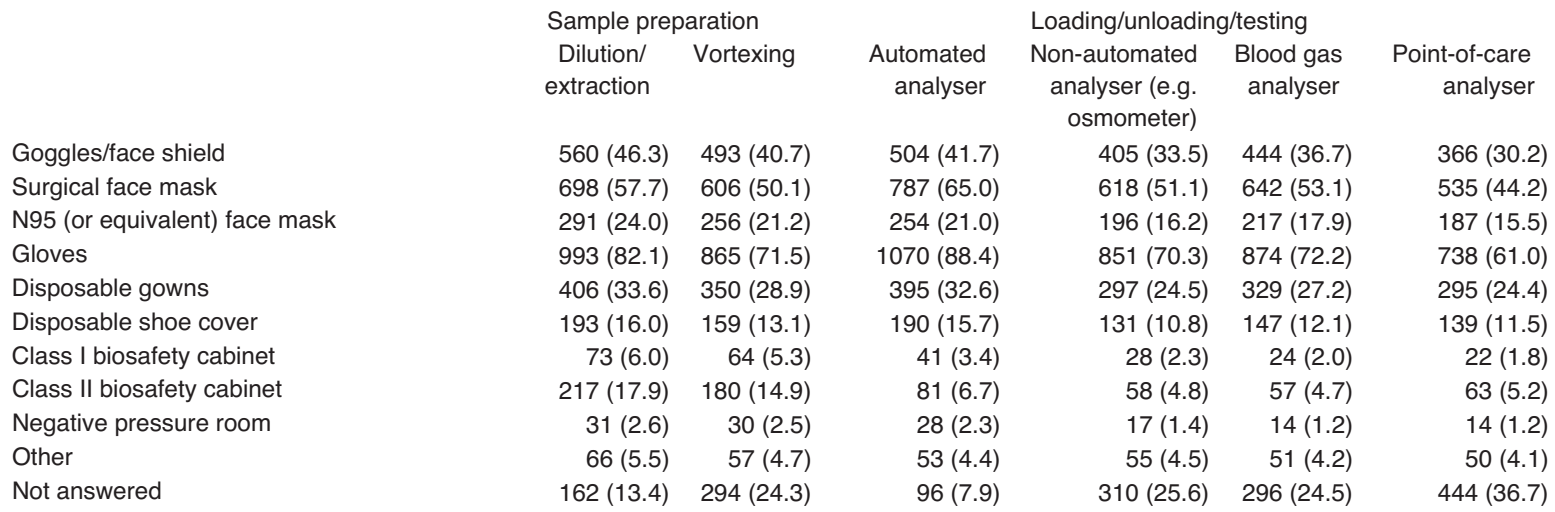

Question: What protection processes do the laboratory staff use when manually performing the following on samples from patients with confirmed COVID-19? Please select all that apply.

\begin{tabular}{|c|c|c|c|c|c|c|}
\hline & Sample pre & paration & & Loading/unloadin & g/testing & \\
\hline & $\begin{array}{l}\text { Dilution/ } \\
\text { extraction }\end{array}$ & Vortexing & $\begin{array}{r}\text { Automated } \\
\text { analyser }\end{array}$ & $\begin{array}{r}\text { Non-automated } \\
\text { analyser (e.g. } \\
\text { osmometer) }\end{array}$ & $\begin{array}{r}\text { Blood gas } \\
\text { analyser }\end{array}$ & $\begin{array}{r}\text { Point-of-care } \\
\text { analyser }\end{array}$ \\
\hline Goggles/face shield & $541(44.7)$ & $485(40.1)$ & $515(42.6)$ & $423(35)$ & $450(37.2)$ & $372(30.7)$ \\
\hline Surgical face mask & $643(53.1)$ & $573(47.4)$ & $728(60.2)$ & $571(47.2)$ & $584(48.3)$ & $502(41.5)$ \\
\hline N95 (or equivalent) face mask & $313(25.9)$ & $274(22.6)$ & $293(24.2)$ & $236(19.5)$ & $251(20.7)$ & $223(18.4)$ \\
\hline Gloves & $930(76.9)$ & $824(68.1)$ & $1009(83.4)$ & $811(67.0)$ & $820(67.8)$ & $702(58.0)$ \\
\hline Disposable gowns & $404(33.4)$ & $357(29.5)$ & $410(33.9)$ & $317(26.2)$ & $337(27.9)$ & $301(24.9)$ \\
\hline Disposable shoe cover & $198(16.4)$ & $170(14.0)$ & $200(16.5)$ & $140(11.6)$ & $154(12.7)$ & $147(12.1)$ \\
\hline Class I biosafety cabinet & $80(6.6)$ & $67(5.5)$ & $46(3.8)$ & $29(2.4)$ & $27(2.2)$ & $22(1.8)$ \\
\hline Class II biosafety cabinet & $211(17.4)$ & $181(15.0)$ & $92(7.6)$ & $62(5.1)$ & $59(4.9)$ & $63(5.2)$ \\
\hline Negative pressure room & $41(3.4)$ & $37(3.1)$ & $32(2.6)$ & $22(1.8)$ & $18(1.5)$ & $25(2.1)$ \\
\hline Other & $68(5.6)$ & $57(4.7)$ & $59(4.9)$ & $56(4.6)$ & $46(3.8)$ & $49(4.0)$ \\
\hline Not answered & $222(18.3)$ & $355(29.3)$ & $152(12.6)$ & $362(29.9)$ & 354 (29.3) & $456(37.7)$ \\
\hline
\end{tabular}

Question: What additional protection processes do the laboratory staff use when manually handling the following on samples from the following patients? Please select all that apply.

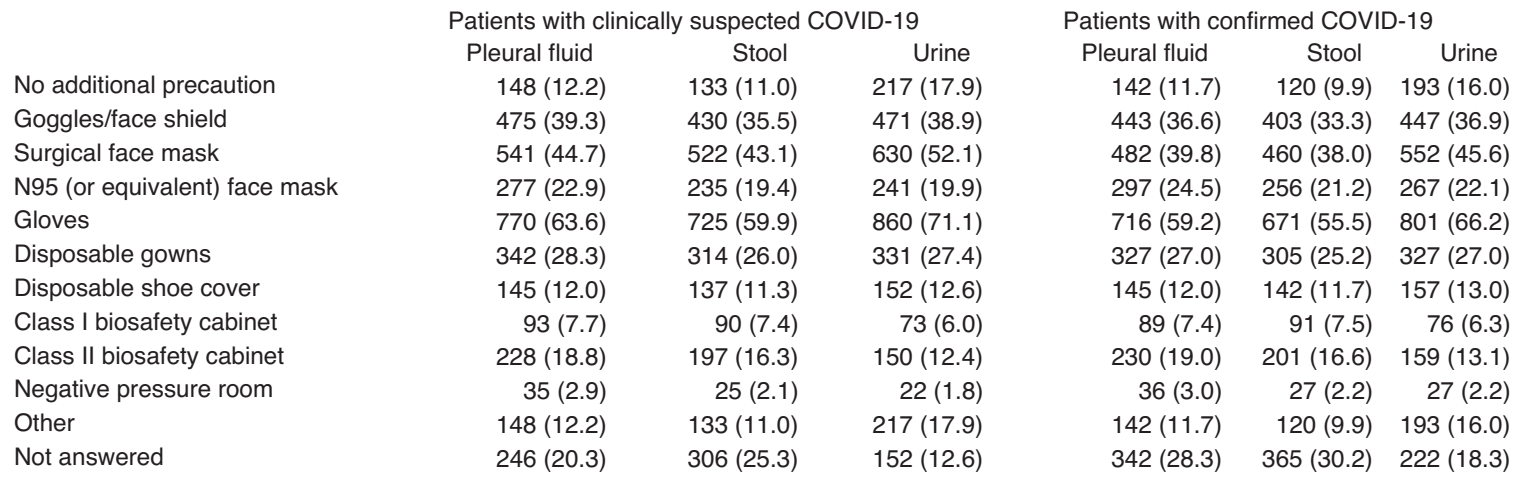

Question: Does your biochemistry laboratory perform additional decontamination step after sample analysis? Please select all that apply.

$\begin{array}{lr}\text { Additional sample probe wash } & 201(16.6) \\ \text { Additional cuvette wash } & 160(13.2) \\ \text { Additional decontamination steps above are performed after every sample } & 201(16.6) \\ \text { Additional decontamination steps above are performed after fixed intervals } & 131(10.8) \\ \text { No additional decontamination step performed } & 689(56.9) \\ \text { Not answered } & 124(10.2)\end{array}$

Figure 2: Frequency of responses to questions related to analytical processes to mitigate biohazard risks in clinical biochemistry laboratories during the COVID-19 pandemic.

The percentages are expressed as fractions of the total number of valid submissions $(n=1210)$. 
sample analysis. A minority (between 10\% and 17\%) of laboratories carried out additional decontamination steps that included an additional sample probe and/or cuvette washes after every sample analysis or at fixed time intervals.

\section{Post-analytical processes}

The responses to the questions regarding post-analytical processes during the COVID-19 outbreak are summarized in Figure 3. A higher number of laboratories recapped clinical samples manually after analysis compared to those which have automated this operation. Similarly, more laboratories switched to keeping patient samples with clinically suspected or confirmed COVID-19 in a standalone fridge/cold room/freezer instead of automated sample storage. There are around one sixth of laboratories that do not recap or store in open bench the samples. About one fifth of laboratories denied add-on testing requests for patients with clinically suspected or confirmed COVID-19. Less than a quarter of laboratories autoclaved clinical samples prior to disposal.

\section{Discussion}

The emergency and rapid spread of COVID-19 pandemic has posed an unprecedented challenge to the laboratory community worldwide. Many laboratories had to respond to the pandemic with limited guidance and large uncertainty concerning the biohazard risks related to the new SARS-CoV-2. This was mainly because the route of transmission and the presence of the virus in various biological fluids were originally unknown or uncertain. The guidelines from professional bodies generally focused on microbiological and molecular biological testing, with little specific guidance for core lab/biochemistry laboratories.

With these initial uncertainties and lack of information, it was very challenging for laboratories to perform

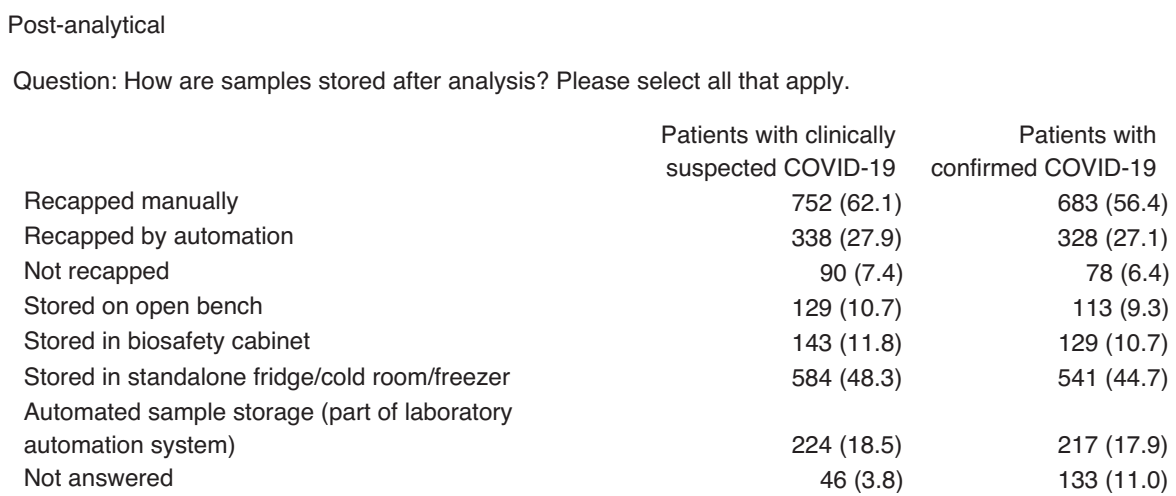

Question: Are add-on testing requests allowed for the following patients?

$\begin{array}{lrr} & \begin{array}{r}\text { Patients with clinically } \\ \text { suspected COVID-19 }\end{array} & \text { Patients with confirmed } \\ \text { COVID-19 }\end{array}$

Question: Are the blood samples of the following patients autoclaved prior to disposal?

$\begin{array}{lrr} & \text { Patients with clinically } & \text { Patients with confirmed } \\ \text { suspected COVID-10 } & \text { COVID-19 } \\ \text { Yes } & 290(24.0) & 276(22.8) \\ \text { No } & 850(70.2) & 787(65.0) \\ \text { Not answered } & 70(5.8) & 147(12.1)\end{array}$

Figure 3: Frequency of responses to questions related to post-analytical processes to mitigate biohazard risks in clinical biochemistry laboratories during the COVID-19 pandemic.

The percentages are expressed as fractions of the total number of valid submissions $(n=1210)$. 
adequate and clinically sound (or evidence-based) biohazard/biosafety risk assessment. This, in turn, hampered their ability to develop and operationalize biohazard protection and precautions within the laboratories. The rapidly evolving epidemic within the community places additional pressure and the consequent change in public health responses also adds challenge to laboratory operations. The limited availability of PPE also limits biosafety precaution practices in many laboratories.

Against the challenges described earlier, the responses of this IFCC survey provide a snapshot of how laboratories are mitigating the biosafety risks of COVID-19 within laboratories that are operating in a rapidly changing and often resource-constraint environment. The key risks while handling clinical samples of COVID-19 patients come from surface contamination on sample container and aerosolization during the pre-analytical and analytical phases (e.g. due to vortexing or sample spillage). As such, PPE and biosafety measures should be applied to minimize such exposures. In this respect, the IFCC Task Force for COVID-19 has just published some biosafety recommendations for clinical laboratories managing samples infected or potentially infected by SARS-CoV-2 [11].

We can hence compare the responses of this survey with some of the IFCC indications. For example, the IFCC Task Force on COVID-19 recommends that all clinical samples of COVID-19 patients be placed in a single layer of (sample/kangaroo) plastic bag, so that the use of additional layers of sample bags used by up to $40 \%$ of laboratories can be considered an unnecessary and even uselessly expensive practice. Moreover, placing the clinical samples in a solid-walled, leak proof container (e.g. cryo-box) has been recommended by the IFCC Task Force on COVID-19, but only approximately half of laboratories that transport samples by hand declared to follow this practice. Notably, pneumatic tube transport has been considered acceptable by the IFCC Task Force on COVID-19 for appropriately sealed specimen containers, but it is not recommended for respiratory samples that have the highest potential viral load.

During the COVID-19 pandemic, it is often difficult to discriminate clinical samples that are collected from infected patients. As such, it is advisable to use face masks, disposable gloves, protective eye wear and laboratory coat or gown when handling clinical samples. A disposable laboratory gown is especially indicated when managing highly contagious specimens such as respiratory samples. It is important to remember that the "simple" surgical face masks, those mostly used according to the survey, guarantee only a limited protection to those wearing them. According to the results of this survey, most laboratory staff do not wear masks, eye wear or disposable gowns other than gloves, even when handling pleural fluids. The use of N95 (or equivalent) masks was considered to be advisable by the IFCC Task Force on COVID-19 when performing aerosol-generating procedures, but this was only practiced by $<20 \%$ of the responders of this survey, even when handling pleural fluid samples that have been shown to have a high viral load. Similarly, the use of level II biosafety cabinet, which can further reduce the risk of aerosolized exposure, was only used in $<20 \%$ of all laboratories that participated in this survey. The lack of biosafety awareness of laboratory personnel might contribute to the currently suboptimal PPE use. Efforts should be directed at educating their importance among clinical laboratory practitioners. Thus, we also recommend that directors of clinical laboratory emphasize the significance through establishing the biosafety team and training laboratory personnel online.

According to various guidance documents, laboratories should preferably use automated instruments and analyzers with closed pre-analytical robotics, and they should also minimize the number of manual processes when handling blood, urine and body fluids. However, the responses from the survey suggested an increased use of manually intensive activities (e.g. recapping, storage), even where automation was available. Although there are no specific guidelines for decontamination of analytical instrumentation after analysis of samples from COVID-19 patients, approximately 15\% of laboratories established additional decontamination steps. These practices may have significant consequences on throughput and turnaround time, and should hence be carefully considered. High-risk specimens, such as respiratory samples, should be disinfected or autoclaved soon after analysis. Nevertheless, only a quarter of all responding laboratories declared to autoclave blood samples prior to disposal.

There are several limitations to this study. Despite the high number of responses received from a representative number of countries and regions, there is under-representation of resource-limited economies. The design of the survey does not mandate answering of all questions. This may have resulted in inadvertent missing responses. Finally, the responses provided are likely to reflect a snapshot of global practices at the time of survey and may evolve over time.

\section{Conclusions}

The responses to this official IFCC survey showed wide variation in pre-analytical, analytical and post-analytical practices in terms of PPE adoption and biosafety processes during the COVID-19 pandemic. It is likely that many of the suboptimal biosafety practices are related to practical local factors, such as limited availability of PPE 
and lack of automated instrumentation. It is important to promote biosafety protective measures and awareness among laboratory personnel. The recently published IFCC guidelines provide specific recommendations for safe laboratory practices in clinical laboratories, and may support the preparation of the staff for managing this and other similar outbreaks in the future. Where possible, these recommendations should be adopted to maintain the safety of essential laboratory staff and their close contacts, thus decreasing the risk of professionally acquired infections.

Acknowledgments: We are grateful to Silvia Colli-Lanzi and Paola Bramati in the IFCC Office in Milan, Kevin Carpenter (Australasian Association for Clinical Biochemistry and Laboratory Medicine), Ana-Maria Simundic (Clinical Chemistry and Laboratory Medicine), Max Reed (New Zealand), Soo-Youn Lee (Korean Society for Clinical Chemistry), Eric Kilpatrick, Chung Shun Ho and Jeffrey Kwok (Hong Kong Society of Clinical Chemistry) for their assistance in disseminating the surveys. We thank Bernard Gouget and Eduardo Luis Freggiaro for helping with the French and Spanish translation of the survey, respectively. We also thank the pilot laboratories for providing valuable feedback that improved the content of the survey. Finally, we thank all the survey participants for taking their time and effort in providing their response to the survey.

Research funding: None declared.

Author contributions: All authors have accepted responsibility for the entire content of this manuscript and approved its submission.

Competing interests: Authors state no conflict of interest.

\section{References}

1. Zhu N, Zhang D, Wang W, Li X, Yang B, Song J, et al. A novel coronavirus from patients with pneumonia in China, 2019. N Engl J Med 2020;382:727-33.
2. World Health Organization. WHO Director-General's opening remarks at the media briefing on COVID-19 - 11 March 2020. https://www.who.int/dg/speeches/detail/who-directorgeneral-s-opening-remarks-at-the-media-briefing-on-covid19---11-March-2020. Accessed: 1 May 2020.

3. Lippi G, Plebani M. The critical role of laboratory medicine during coronavirus disease 2019 (COVID-19) and other viral outbreaks. Clin Chem Lab Med 2020;58:1063-9.

4. Binnicker MJ. Emergence of a novel coronavirus disease (COVID19) and the importance of diagnostic testing: why partnership between clinical laboratories, public health agencies, and industry is essential to control the outbreak. Clin Chem 2020;66:664-6.

5. World Health Organization. Laboratory biosafety guidance related to coronavirus disease 2019 (COVID-19): interim guidance, 12 February 2020. https://apps.who.int/iris/handle/10665/331138. Accessed: 1 May 2020.

6. Centers for Disease Control and Prevention. Interim laboratory biosafety guidelines for handling and processing specimens associated with coronavirus disease 2019 (COVID-19). https:// www.cdc.gov/coronavirus/2019-nCoV/lab/lab-biosafety-guidelines.html. Accessed: 1 May 2020.

7. Hong KH, Lee SW, Kim TS, Huh HJ, Lee J, Kim SY, et al. Guidelines for laboratory diagnosis of coronavirus disease 2019 (COVID-19) in Korea. Ann Lab Med 2020;40:351-60.

8. Hawkins R. Preparing the biochemistry laboratory for the next outbreak: lessons from SARS in Singapore. Clin Biochem Rev 2005;26:59-64.

9. Zheng S, Fan J, Yu F, Feng B, Lou B, Zou Q, et al. Viral load dynamics and disease severity in patients infected with SARSCoV-2 in Zhejiang province, China, January-March 2020: retrospective cohort study. Br Med J 2020;369:m1443.

10. Loh TP, Horvath AR, Wang C-B, Koch D, Adeli K, Mancini N, et al. Operational considerations and challenges of biochemistry laboratories during the COVID-19 outbreak: an IFCC global survey. Clin Chem Lab Med 2020;58:1441-9.

11. Lippi G, Adeli K, Ferrari M, Horvath AR, Koch D, Sethi S, et al. Biosafety measures for preventing infection from COVID-19 in clinical laboratories: IFCC Taskforce Recommendations. Clin Chem Lab Med 2020;58:1053-62.

Supplementary offers supplementary material (https://doi.org/ 10.1515/cclm-2020-0711). 